\title{
Uma Análise baseada em Clusterização para Monitoramento Integrado da Atividade da Pesca Artesanal na Bacia Araguaia-Tocantins
}

\author{
Renato S. da Silva ${ }^{1,2}$, Rogério R. da Silva ${ }^{1}$, Hugo P. Kuribayashi ${ }^{2}$, \\ Cristiane V. da Cunha ${ }^{3,4}$, Keid Nolan S. Sousa ${ }^{4}$ \\ ${ }^{1}$ Laboratório de Computação Científica (LCC) \\ Universidade Federal do Sul e Sudeste do Pará (Unifesspa) \\ Caixa Postal 100 - 66.507-590 - Marabá - PA - Brasil \\ ${ }^{2}$ Instituto de Geociências e Engenharias (IGE) \\ Universidade Federal do Sul e Sudeste do Pará (Unifesspa) - Marabá, PA - Brasil \\ ${ }^{3}$ Instituto de Ciências Humanas (ICH) \\ Universidade Federal do Sul e Sudeste do Pará (Unifesspa) - Marabá, PA - Brasil \\ ${ }^{4}$ Programa de Pós-Graduação em Sociedade, Natureza e Desenvolvimento (PPGSND) \\ Universidade Federal do Oeste do Pará (UFOPA) - Santarém, PA - Brasil \\ \{renatosabino,rromulo, hugo,crisvieira_cunha\}@unifesspa.edu.br \\ keid.ufopa@gmail.com
}

\begin{abstract}
This work presents an Adaptive Fisheries Monitoring Model that was evaluated by the method of clustering fishery data collected in 2016-2017 in the Araguaia-Tocantins basin. Data were obtained from the Integrated Fish Statistics System (SIEPE), which generated categorical and numerical variables. The analysis reveals the most significant numerical variables in the study were the "fishing yield" and "engine power" of the watercraft, while the most expressive categorical variables were "popular species name" and "fishing environment". These variables should be taken into account in fisheries monitoring programs in the Araguaia-Tocantins basin, as well as the use of SIEPE to support fisheries management at different scales.
\end{abstract}

Resumo. Este trabalho apresenta um modelo de Monitoramento Adaptativo da Pesca que foi avaliado pelo método de clusterização de dados de pesca coletados em 2016-2017 na bacia Araguaia-Tocantins. Os dados foram obtidos do Sistema Integrado de Estatística Pesqueira (SIEPE), que gerou variáveis categóricas e numéricas. A análise revela que as variáveis numéricas mais expressivas no estudo foram o rendimento da pesca e potência do motor da embarcação, enquanto que as variáveis categóricas mais expressivas foram, "nome popular espécie" e "ambiente de pesca". Estas variáveis devem ser levadas em consideração em programas de monitoramento da pesca na bacia Araguaia-Tocantins, bem como o uso do SIEPE para apoiar a gestão pesqueira em diferentes escalas. 


\section{Introdução}

As pescarias continentais mundiais produziram em 2012 cerca de 11 milhões de toneladas de peixe, que representam elevada importância devido à sua contribuição econômica e social para o rendimento das comunidades rurais [FAO 2016]. Em países em desenvolvimento, a pesca de pequena escala representa grande importância para a seguridade alimentar e econômica [Kolding et al. 2014]. No contexto mundial, o Brasil está entre os maiores produtores de pescado, com 235 mil toneladas de peixes desembarcados em 2014, posicionado na $11^{\mathrm{a}}$ posição no ranking mundial [FAO 2016]. No entanto estes dados possivelmente são subestimados ou imprecisos pois são desatualizados e há escassez de recursos e ferramentas para a coleta de informações [Oviedo and Bursztyn 2017, Kolding et al. 2014].

$\mathrm{Na}$ Amazônia brasileira, programas de monitoramento da pesca em escala local ou regional são desenvolvidos nos últimos 50 anos de forma descontinuada e com diferentes abordagens metodológicas, como censo diário em portos de desembarque pesqueiro, pescarias experimentais, estimativas de pesca por entrevistas e abordagens participativas, incluindo o uso de aplicativos em telefones móveis [Barthem et al. 1991, Batista et al. 1998, Cetra and Petrere 2001, Isaac et al. 2008, Hallwass 2015, Oviedo and Bursztyn 2017]. Dentre as metodologias utilizadas em programas de monitoramento na Amazônia as que tiveram melhor desempenho foram as baseadas na participação social. A exemplo, citamos o projeto ProVárzea/Ibama, que também avançou em instrumentos de armazenamento de informações sobre a pesca por meio do Sistema Integrado de Estatística Pesqueira para a Amazônia [Ruffino 2008]. Nas demais regiões da Amazônia as iniciativas de integração de dados sobre a pesca ainda não são uma realidade.

Portanto, este artigo tem como objetivo apresentar uma análise baseada em técnicas não supervisionadas de machine learning, a partir de algoritmos de clusterização para sumarização e extração de padrões e agrupamentos dos dados, de modo a fornecer informações complementares à estatística pesqueira tradicional. A abordagem utilizada tomou como base, dados do Sistema Integrado de Estatística Pesqueira (SIEPE), que foi desenvolvido para atender o Programa de Monitoramento Adaptativo da Pesca na média bacia Araguaia-Tocantins. Os dados utilizados do SIEPE foram coletados no Monitoramento Adaptativo da Pesca realizado entre setembro 2016 a outubro de 2017 nas comunidades ribeirinhas de Itupiranga e São Geraldo do Araguaia, na bacia Araguaia Tocantins, Pará, Brasil.

Como organização do trabalho, na seção 2 são apresentados os materiais e métodos utilizados na pesquisa, dentre eles a descrição do processo de Monitoramento Adaptativo de Pesca, uma breve discussão sobre métodos de clusterização de dados mistos utilizados e o projeto e arquitetura do SIEPE. Na seção 3 são apresentados os resultados e discussão dos dados analisados por meio de técnicas de clusterização; e a seção 4 apresenta as considerações finais e discussões sobre trabalhos futuros.

\section{Métodos}

\subsection{Monitoramento Adaptativo Pesqueiro}

O modelo de Monitoramento Adaptativo da Pesca (MAP) foi realizado entre os anos de 2015 a 2019. Este modelo buscou compreender a complexidade do sis- 
tema ecológico da porção média da bacia Araguaia-Tocantins adotando múltiplas metodologias com capacidade para evoluir à medida que novos problemas fossem surgindo [Lindenmayer and Likens 2009, Lindenmayer et al. 2011]. O objetivo central nos modelos de monitoramento adaptativo é proporcionar que as informações coletadas possam gerar políticas públicas e evoluir para programas de manejo adaptativo [Lindenmayer and Likens 2009, Lindenmayer et al. 2011]. O MAP foi realizado nas comunidades ribeirinhas de Santa Teresinha do Tauiri e Santo Antoninho, situadas às margens do rio Tocantins em Itupiranga/PA e nas comunidades de Santa Cruz dos Martírios e Ilha de Campo, localizadas às margens do rio Araguaia em São Geraldo do Araguaia/PA. As comunidades estão localizadas na porção média da bacia Araguaia-Tocantins, conforme divisão sugerida em [MMA 2006].

Para realização do MAP foram consideradas 03 (três) etapas: 1. Construção de confiança: que incluiu estabelecer parcerias dentro das comunidades, entre instituições e pesquisadores para alinhamento coletivo dos objetivos e metodologia do monitoramento participativo da pesca e capacitação dos pescadores em processo contínuo que iniciou em 2015. 2. Monitoramento Participativo: foi desenvolvido entre setembro de 2016 a outubro de 2017 e consistiu na aplicação do monitoramento da pesca, planejamentos coletivos e estruturação de um sistema (SIEPE), que pudesse armazenar e tratar as informações coletadas. 3. Consolidação: consistiu no desenvolvimento, teste funcional e avaliação do MAP e do SIEPE.

O monitoramento seguiu metodologia adaptada de [Hallwass 2015], onde cada pescador voluntário preencheu cartões de desembarque de 05 (cinco) pescarias do mês durante 01 (um) ano. As informações preenchidas nos cartões de desembarque continham os seguintes atributos: rio, data, pescador, quantidade de pescadores na pescaria, tempo gasto na pescaria (horas), embarcação utilizada, nome do local da pesca, ambiente, petrecho utilizado, tamanho e quantidade de redes, nome popular dos cinco principais peixes capturados, captura em $\mathrm{kg}$ por peixe, captura em $\mathrm{kg}$ total, $\mathrm{kg}$ vendidos, $\mathrm{kg}$ consumidos, total recebido ( $\mathrm{R} \$$-Reais), custos da pescaria ( $\mathrm{R} \$$-Reais).

Os cartões de desembarques foram entregues a 25 pescadores que se voluntariaram a participar da pesquisa. Os pescadores foram capacitados para o preenchimento dos cartões, que após preenchidos foram recolhidos pelos pesquisadores e as informações digitadas inicialmente em planilhas eletrônicas que foram importadas para o SIEPE. A captura por unidade de esforço (CPUE) foi utilizada para a avaliação das pescarias seguindo adaptações de [Petrere Jr 1978]. Para garantir a análise da CPUE, os únicos atributos que não puderam ficar sem preenchimento no SIEPE foram: rio, data, pescador, tempo gasto na pescaria (horas), quantidade de pescadores envolvidos na pesca e captura em $\mathrm{kg}$ total. Estas informações são consideradas essenciais pois dão subsídio para a estatística pesqueira amplamente utilizada por pesquisadores na Amazônia [Ruffino 2004, Petrere Jr and Peixer 2007, Hallwass and Silvano 2015, Zacarkim et al. 2015, da Silva Jr. et al. 2017].

\subsubsection{Arquitetura e Modelagem de Dados do SIEPE}

A arquitetura proposta para o SIEPE está baseada na adoção de plataformas Web e permite o acesso por meio de navegadores. Possui vantagens, como a heterogeneidade de plata- 
formas e dispositivos. Adota tecnologias diversas, que provê melhor portabilidade e acessibilidade para os usuários do sistema. Entre as metodologias utilizadas para construção do sistema podemos destacar a eXtreme Programming (XP). As ferramentas utilizadas foram: PHP, PostgreSQL, Servidor Web Apache e Selenium. Testes funcionais foram realizados por meio do framework Selenium com codificação em linguagem Java.

O SIEPE possui uma arquitetura baseada em MVC (Model-View-Controller). O Model, consiste nos dados da aplicação, regras de negócios, lógica e funções. A View, é qualquer saída de representação dos dados, como uma tabela ou um diagrama. O Controller, gerencia o fluxo de dados entre os componentes do sistema. O SIEPE ainda adota o padrão arquitetural de acesso de dados DAO (Data Access Object) e as suas funcionalidades estão organizadas em: Registrar Pesca, Gerenciar Cadastros Auxiliares da pesca, Importar Dados, Realizar Consulta, Gerar Relatórios, Visualizar Dados da Pesca e Exportar Dados. Este sistema, promove o armazenamento, tratamento e correlacionamento dos dados do monitoramento da pesca. Tem potencial de integrar informações de diversas bacias pesqueiras para possibilitar análise da dinâmica da pesca de diferentes escalas.

\subsubsection{Clusterização de Dados Mistos}

O particionamento do conjunto de dados em grupos homogêneos ou clusters é uma operação fundamental do processo de análise de dados. É uma operação útil em diferentes situações, como classificação (não supervisionada), agregação, segmentação, por exemplo. Clusterização é uma abordagem comum na literatura, para particionamento de conjuntos de dados, de tal forma que os objetos classificados sob um mesmo cluster apresentam maior similaridade entre si, em relação à objetos de outros agrupamentos [Huang 1998].

Em termos de clusterização a literatura apresenta diversas abordagens de agrupamento, relacionados à dados numéricos e categóricos. Em ambos os casos é desejável a adoção de técnicas que realizem tal agrupamento de modo eficiente, em geral para grandes bases de dados. Como os dados apresentados no SIEPE consistem de dados numéricos e dados categóricos, o algoritmo a ser utilizado deveria ser capaz de trabalhar de modo eficiente com os ambos tipos de dados. Assim, durante a análise em questão optou-se pela utilização do algoritmo $k$-prototypes [Huang 1998]. Este algoritmo combina a capacidade de agrupamento de dados numéricos do algoritmos k-means [Anderberg 1973], com o algoritmo k-modes, que realiza agrupamento de dados categóricos por meio da medição de dissimilaridade entre os objetos.

Assim, dado um conjunto de dados $\mathrm{X}$, contendo $\mathrm{n}$ objetos de dados descritos por $m_{r}$ atributos numéricos e $m_{c}$ atributos categóricos, o objetivo do algoritmo k-prototypes é minimizar a seguinte função objetivo:

$$
J=\sum_{l=1}^{k} \sum_{i=1}^{n} p_{i l} d\left(x_{i}, Q_{l}\right)
$$

onde $p_{i l}$ é uma variável binária, isto é $p_{i l} \in\{0,1\}$, indicando a pertinência do objeto de dados $x_{i}$ no cluster $l$; $Q_{l}$ é o prototype (centro) do cluster $l$ e $d\left(x_{i}, Q_{l}\right)$ é a 
dissimilaridade medida, que é definida como:

$$
d\left(x_{i}, Q_{l}\right)=\sum_{r=1}^{m_{r}}\left(x_{i r}-q_{l r}\right)^{2}+\gamma_{l} \sum_{c=1}^{m_{c}} \delta\left(x_{i c}, q_{l c}\right),
$$

onde $x_{i r}$ representa o valores dos atributos numéricos e $x_{i c}$ o valores dos atributos categóricos para cada objeto de dados $x_{i} . Q_{l}=q_{l 1}, \ldots, q_{l m}$ representa os centroides do cluster 1 , onde $q_{l r}$ é a média do atributo numérico $r$ e do cluster $l$, enquanto $q_{l c}$ é a amostra mais comum (moda) do atributo categórico $c$ e cluster $l$. Para atributos categóricos, $\delta(p, q)=0$ para $p \equiv q$ e $\delta(p, q)=1$ para $p \neq q$. $\gamma_{l}$ é um peso para atributos categóricos, de modo a balancear a influência entre as componentes da equação. A formulação geral do algoritmo k-prototypes é descrita pelo algoritmo 1.

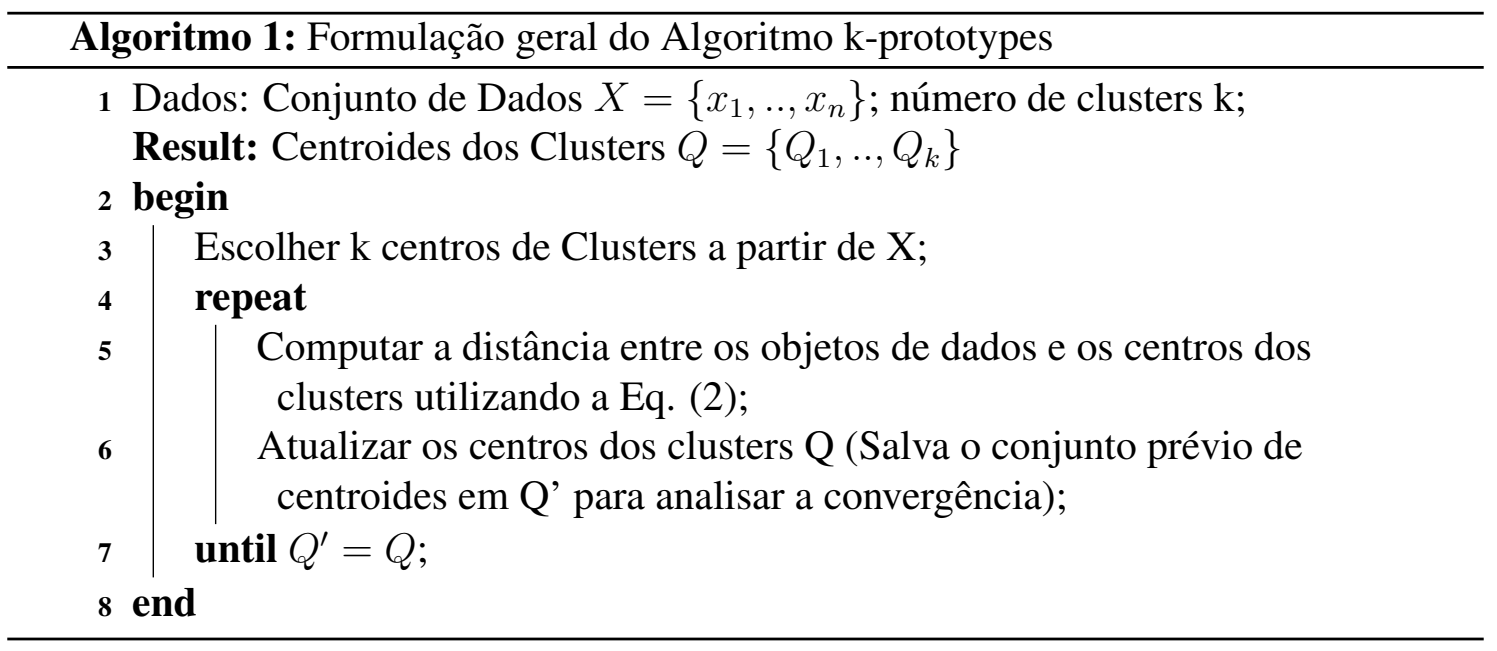

\section{Análise de Dados e Resultados}

O desenvolvimento do MAP registrou 477 desembarques de pesca entre setembro de 2016 à outubro de 2017 que foram incluídos no SIEPE. O SIEPE permitiu a inclusão de 100\% das amostras do MAP e não foram observados dados ausentes ou que necessitassem de transformação. Todas as funcionalidades da arquitetura do SIEPE tiveram bom funcionamento. A exportação das informações de pesca do SIEPE foi realizada com as atividades de pré-processamento de dados e gerou múltiplas variáveis categóricas e numéricas.

A organização dos dados relacionados é composta por um total de 09 (nove) variáveis categóricas (Comunidade, Local de Pesca, Cidade, Rio, Descrição da Embarcação, Descrição da Arte de Pesca, Descrição do Comprador, Ambiente de Pesca e Nome Popular da Espécie) e 08 (oito) variáveis numéricas (Dia da pesca, Mês da pesca, Número de Pescadores, Peso Consumido, Peso Vendido, Valor de Custo da Pesca, Valor de Venda da Pesca e Potência da Embarcação).

Nesta análise foi realizado um estudo de percentual de variação em função do número de clusters. Para este estudo foram considerados de 01 a 08 clusters inicialmente, e buscou-se a adoção do número de clusters que minimizassem a soma das áreas do clusters e ainda assim fornecessem melhor modelagem de dados. Nesse contexto o ganho marginal foi registrado como significativo a partir de 03 (três) clusters, conforme flexão 
na função do percentual de variabilidade, valor este que foi ratificado conforme análise dos centroides obtidos por meio do algoritmo k-prototypes.

Para execução do algoritmo $k$-prototypes, utilizou-se como parâmetros $\gamma_{l}=0.1$, e com base em sua execução observou-se que os dados classificaram-se em 03 (três) grupos distintos (Figura 1). Em relação às amostras, 338 foram classificadas no cluster 1, 07 no cluster 2 e 132 amostras foram agrupadas no cluster 3 . Os clusters encontrados possuem características similares ao se considerar parte dos atributos categóricos. No entanto, ao se considerar os centroides dos clusters 1,2 e 3, em especial a variável numérica, valor de venda do pescado, observa-se que os clusters obtiveram valores expressivamente distintos, indicando uma possível finalidade da atividade pesqueira monitorada.

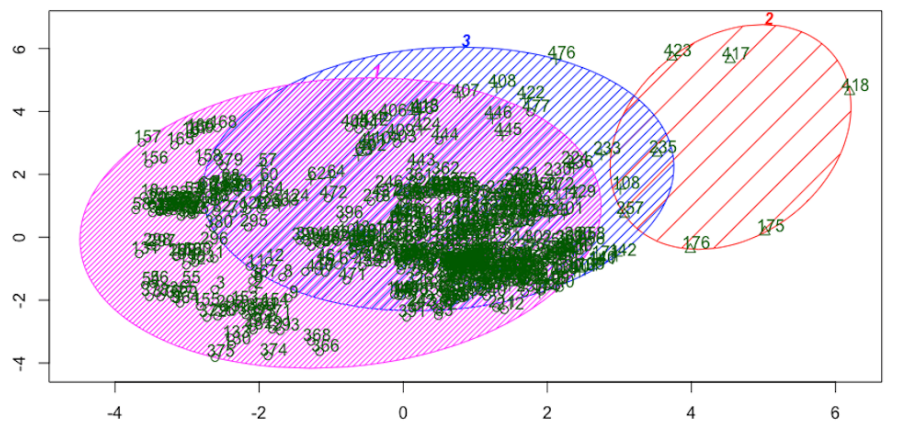

Figura 1. Representação gráfica em 2D demonstrando agrupamento em cluster de dados numéricos e categóricos do SIEPE

As variáveis numéricas foram as que mais influenciaram na análise, em especial a variável valor de venda e potência da embarcação. As variáveis categóricas que mais influenciaram foram nome popular espécie e ambiente de pesca. A variável que representa o valor de venda da pesca foi responsável por cerca de 33,98\% da variabilidade dos dados, além de representar a variável que melhor divide os dados em questão, conforme dados apresentados pela Tabela 1.

Em adição ao exposto, a variável valor de venda apresenta valores bem característicos para cada um dos clusters apresentados. É importante destacar que para o cluster 2 , o primeiro quartil e a mediana possuem valores aproximados de $\mathrm{R} \$ 1.000,00$, enquanto que para o cluster 3, estes valores aproxima-se de $\mathrm{R} \$ 400,00$. Para melhor explicar a diferença financeira entre os clusters, buscou-se observar inicialmente outras variáveis numéricas, como número de pescadores, peso consumido e potência da embarcação. Para a variável potência da embarcação, observa-se que o cluster 2 apresenta como valor, 7,57 (hp), indicando que as embarcações utilizadas por pescadores agrupados no cluster 2 , são de maior porte do que as do cluster 1 e 3 (Tabela 1).

Pescarias com barcos motorizados em geral apresentam maior quantidade de peixes capturados, como já observado no médio Araguaia [da Silva and de Farias 2017], no baixo Tocantins [Hallwass et al. 2013] e outras regiões da Amazônia [Isaac et al. 2008]. O comportamento do pescador (número de pescadores e potência da embarcação), aqui analisado, foi capaz de influenciar em diferenças no rendimento da pesca artesanal. Esta constatação pode estar relacionada à necessidade dos pescadores, que tem motores mais potentes, necessitarem aumentar o esforço, devido ao custo mais elevado com petrechos de pesca e combustível, que pode estar relacionado com a escolha de espécies alvos. 
Tabela 1. Dados de centroides encontrados por meio do k-prototypes

\begin{tabular}{|c|c|c|c|}
\hline & Cluster 1 & Cluster 2 & Cluster 3 \\
\hline Amostras & 338 & 7 & 132 \\
\hline Valor Venda & $\mathrm{R} \$ 66,79$ & $\mathrm{R} \$ 1.161,00$ & $\mathrm{R} \$ 335,93$ \\
\hline N. Pescadores & 1,85 & 2,0 & 2,02 \\
\hline Peso Consumido & $3,12 \mathrm{~kg}$ & $2,57 \mathrm{~kg}$ & $2,39 \mathrm{~kg}$ \\
\hline Potência do Motor & $5,45 \mathrm{hp}$ & $7,57 \mathrm{hp}$ & $5,41 \mathrm{hp}$ \\
\hline Nome Popular Espécie & Tucunaré & $\begin{array}{c}\text { Avoador, Piau, Curimatá, Pacu, } \\
\text { Branquinha, Mapará }\end{array}$ & Avoador \\
\hline Ambiente de Pesca & Pedral, Meio do Rio & Meio do Rio, Beira do Rio & Pedral, Meio do Rio \\
\hline
\end{tabular}

Ao analisar os dados categóricos, em especial, as variáveis nome popular espécie e ambiente de pesca, as diferenças observadas entre os clusters ficam ainda mais evidentes e sugerem que as pescarias na média bacia Araguaia-Tocantins, mesmo sendo multi-específicas [Hallwass and Silvano 2015, Zacarkim et al. 2015], apresentam moderada seletividade para espécies com maior valor comercial, ou maior abundância na bacia, relacionado ainda à escolha do ambiente de pesca.

Com relação à variável nome popular espécie, observa-se que no cluster 1 o principal grupo de peixes capturados é o Tucunaré (Cichla spp., C. kelberi, C. piquiti), em ambientes de pedral e meio do rio. O Tucunaré é considerado um peixe nobre e que representa elevada importância econômica no rio Araguaia [Zacarkim et al. 2015] e no rio Tocantins [Alves and Barthem 2008, Prysthon et al. 2019]. Devido ser um dos peixes de maior valor econômico no Araguaia-Tocantins, espera-se que o rendimento dos pescadores em relação a esta espécie seja alto, no entanto, não foi o observado na análise. Desta forma sugere-se que os pescadores agrupados no cluster 1, estejam direcionando a venda do pescado a atravessadores, que compram por um valor subestimado, fazendo com que o rendimento caia. Situações semelhantes a esta já foram observadas no rio Tocantins e outras regiões da Amazônia, onde o pescador torna-se dependente de um sistema de exploração [de Camargo and Petrere Jr 2004, Fraxe et al. 2007, Petrere Jr and Peixer 2007]. Neste caso, é importante observar se os rendimentos capturados nos clusters 2 e 3 se refere à quantia justa da produção do pescador.

No cluster 2, estão agrupados amostras de pescarias em ambientes no meio do rio e na beira do rio, para captura de grupos de espécies variadas, como Avoador (Hemiodus unimaculatus, $H$. microlepis, $H$. argenteus), Piau (Leporinus spp., Leporinus fasciatus e Schizodon vittatus), Curimatá (Prochilodus nigricans), Pacu (Myleus setiger, Myloplus rubripinnis, Myloplus asterias, Mylesinus paucisquamatus), Branquinha (Curimata spp., C. cyprinoides, C. knerii) e Mapará (Hypophthalmus spp., H. marginatus, H. edentatus, $H$. fimbriatus), que representam espécies de maior expressão comercial ou maior abundância na bacia [Hallwass and Silvano 2015, Zacarkim et al. 2015, Juras et al. 2004, da Silva and de Farias 2017].

A diversidade de espécies capturadas talvez seja o fator mais relevante para as pescarias do cluster 2, garantindo assim, melhores condições de negociação, elevando o rendimento. É importante enfatizar, que a pesca do Avoador e Mapará (espécies mais expressivas nos clusters 2 e 3), representam grande biomassa desembarcada no rio Tocantins [Juras et al. 2004, Hallwass 2011, Prysthon et al. 2019]. Porém, o valor de venda 
desses peixes é de no máximo $\mathrm{R} \$ 2,50 / \mathrm{kg}$ (verificação de campo). Pela grande quantidade capturada, os pescadores necessitam de motores mais potentes para auxiliar na propulsão da embarcação, além de que, os petrechos utilizados em pescarias de Mapará e Avoador, necessitam de alto investimento financeiro. Neste caso, especial atenção deve ser dada ao cluster 2, que apresenta volume financeiro médio superior em torno de $345 \%$ em relação ao cluster 3 . Uma pesquisa mais apurada poderia ser realizada com o propósito de verificar se esses rendimentos estão sendo direcionados para os pescadores, ou para atravessadores, em um modelo de exploração do trabalho.

No cluster 3, o grupo de espécies mais capturada é o Avoador, em ambientes de pedrais e meio do rio. O rendimento deste grupo de pescadores não é tão alto como no cluster 2, e nem a potência dos motores de suas embarcações. É possível dizer que os pescadores que estão investindo na pesca do voador, empregam maior esforço durante a safra desta espécie (junho a setembro), que mesmo tendo valor comercial baixo, compensa pela quantidade capturada. No decorrer do ano o esforço é direcionado para outras espécies, o que explicaria a sobreposição dos clusters 1, 2 e 3 (Figura 1).

Cabe ressaltar, que há similaridades entre os dados observados, em especial ao se considerar os dados numéricos. Entende-se que os clusters 1, 2 e 3 estão relacionados à atividade pesqueira de pequena escala e para subsistência familiar, mesmo que alguns grupos se sobressaiam em relação ao rendimento. A constatação de que o tipo de pescaria é de pequena escala e subsistência, se dá em consideração pela quantidade de pescado consumido por dia de pesca, além da potência do motor das embarcações e a tripulação embarcada [da Silva and de Farias 2017]. Mediante esta constatação, a aplicação de políticas de proteção dos recursos hídricos e pesqueiros representa questão de fundamental importância dos pontos de vista ambiental, da economia local e de seguridade alimentar na bacia Araguaia-Tocantins.

\section{Considerações Finais}

Após o desenvolvimento deste trabalho, que incluiu a implantação de um modelo de Monitoramento Adaptativo da Pesca, a construção de um Sistema Integrado de Estatística Pesqueira e a avaliação da eficiência destas duas ferramentas para gestão pesqueira, podemos considerar: (i) MAP: Tem viabilidade financeira, se comparado aos programas de monitoramento da pesca tradicionais, pois adota como princípio o estabelecimento de parcerias; Tem retorno social; Quando consolidado poderá ser a base para o manejo e gestão adaptativa de ecossistemas altamente alterados e que ainda não tenham iniciativas de monitoramento; $O$ modelo pode ser adotado em locais que já tenham medidas de monitoramento da pesca sem prejuízos aos dados pretéritos coletados; (ii) SIEPE: garantiu um retorno de $100 \%$ das informações de pesca armazenadas, gerando múltiplas variáveis categóricas e numéricas para avaliação do MAP; É um recurso de armazenamento e tratamento de informações da pesca inovador e promissor; Tem capacidade de integrar e relacionar dados pesqueiros de diferentes bacias hidrográficas para apoiar a gestão pesqueira. (iii) Método de Clusterização de Dados Mistos: Foi capaz de expressar uma visão mais atualizada sobre o rendimento da pesca na bacia Araguaia-Tocantins; Indicou padrões por meio do agrupamento de dados utilizando variáveis numéricas e categóricas; Indicou que há pressão de pesca pelo Mapará, voador e tucunaré, desta forma são necessárias medidas de manejo para estes grupos de espécies; A potência do motor da embarcação influencia no rendimento da pesca e deve ser levada em consideração nos planos de manejo para a 
bacia Araguaia-Tocantins.

Em relação a trabalhos futuros, consideramos que o desenvolvimento de programas de monitoramento da pesca na bacia Araguaia-Tocantins, devem levar em consideração as variáveis que se destacaram neste estudo: valor de venda do pescado, potência do motor da embarcação, peixes capturados e ambientes de pesca, como adicional a estatística pesqueira tradicional. Para o melhor funcionamento do SIEPE é necessário relacionar o sistema a aplicativo de monitoramento de pesca em dispositivo móvel; Construir modelo de estatística básica para analisar os dados armazenados no SIEPE; e Relacionar os dados da pesca com recursos de geoprocessamento, de modo apoiar a gestão pesqueira em diferentes escalas.

\section{Referências}

Alves, M. C. B. and Barthem, R. B. (2008). A Pesca Comercial dos "Tucunarés"Cichla spp. (Perciformes, Cichlidae) no Reservatório da UHE-Tucuruí, Rio Tocantins, PA. Inst. Pesca, 34(4):553-561.

Anderberg, M. R. (1973). Cluster analysis for applications. Probability and mathematical statistics, 19 CN - QA278.A5 1973.

Barthem, R. B., de Brito Ribeiro, M. C. L., and Petrere, M. (1991). Life strategies of some long-distance migratory catfish in relation to hydroelectric dams in the Amazon Basin. Biological Conservation, (55):339-345.

Batista, V. S., Inhamuns, A. J., Freitas, C. E. C., and Freire-Brasil, D. (1998). Characterization of the fishery in river communities in the low-Solimões/high-Amazon region. Fisheries Management and Ecology, 5(5):419-435.

Cetra, M. and Petrere, M. (2001). Small-scale fisheries in the middle River Tocantins, Imperatriz (MA), Brazil. Fisheries Management and Ecology, (8):53-162.

da Silva, A. P. and de Farias, E. G. G. (2017). Caracterização participativa da frota pesqueira do Rio Araguaia - Tocantins, Brasil. Magistra, 29(1):80-90.

da Silva Jr., U. L., Raseira, M. B., Ruffino, M. L., Batista, V. d. S., and Leite, R. G. (2017). Estimativas do Tamanho do Estoque de algumas Espécies de Peixes Comerciais da Amazônia a partir de Dados de Captura e Esforço. Biodiversidade Brasileira, $7(1): 105-121$.

de Camargo, S. A. F. and Petrere Jr, M. (2004). Análise de risco aplicada ao manejo precaucionário das pescarias artesanais na região do reservatório da UHE-Tucuruí (Pará, Brasil). Acta Amazonica, 34(3):473-485.

FAO (2016). The State of World Fisheries and Aquaculture 2016. Contributing to food security and nutrition for all. Technical report.

Fraxe, T. d. J. P., Witkoski, A. C., and Pereira, H. d. S. (2007). Comunidades ribeirinhas amazônicas: memória, ethos e identidade. Manaus: EDUA, page 224.

Hallwass, G. (2011). Ecologia Humana da Pesca e Mudanças Ambientais no Baixo Rio Tocantins , Amazônia Brasileira.

Hallwass, G. (2015). Etnoecologia e Pesca: Influência de unidades de conservação e aplicação do conhecimento ecológico local de pescadores no manejo e conservação 
dos recursos pesqueiros no Baixo Rio Tapajós, Amazônia brasileira. PhD thesis, Universidade Federal do Rio Grande do Sul.

Hallwass, G. et al. (2013). Behavioral and environmental influences on fishing rewards and the outcomes of alternative management scenarios for large tropical rivers. Journal of Environmental Management, (128):274-278.

Hallwass, G. and Silvano, R. A. M. (2015). Patterns of selectiveness in the Amazonian freshwater fisheries: implications for management. Journal of Environmental Planning and Management, 59(9):1537-1559.

Huang, Z. (1998). Extensions to the k-means algorithm for clustering large data sets with categorical values. Data Mining and Knowledge Discovery.

Isaac, V. J., Da Silva, C. O., and Ruffino, M. L. (2008). The artisanal fishery fleet of the lower Amazon. Fisheries Management and Ecology, (15):179-187.

Juras, A. A., Cintra, I. H. A., and Ludovino, R. M. R. (2004). A pesca na área de influência da usina hidrelétrica de tucuruí, estado do pará. Boletim técnico-científico do cepnor. 4(1):77-88.

Kolding, J., Béné, C., and Bavinck, M. (2014). Small-scale fisheries, chapter 22, pages 317-331. John Wiley \& Sons, Ltd.

Lindenmayer, D. B. and Likens, G. E. (2009). Adaptive monitoring: a new paradigm for long-term research and monitoring. Trends in Ecology and Evolution.

Lindenmayer, D. B., Likens, G. E., Haywood, A., and Miezis, L. (2011). Adaptive monitoring in the real world: Proof of concept.

MMA (2006). Programa de Estruturação Institucional para Consolidação da Política Nacional de Recursos Hídricos - BRA/OEA/01/002. Technical report, Ministério do Meio Ambiente, Brasília-DF.

Oviedo, A. F. P. and Bursztyn, M. (2017). Decentralization and Fisheries Management in The Brazilian Amazon: Resource Rights and Accountability. Ambiente \& Sociedade.

Petrere Jr, M. (1978). Pesca e esforço de pesca no Estado do Amazonas I - Esforço e captura por unidade de esforço. Acta Amaz, 8(3):439-454.

Petrere Jr, M. and Peixer, J. (2007). O setor pesqueiro na amazônia: situação atual e tendências. Ibama/PróVárzea, 122 pp.

Prysthon, A., Cunha, V. C., and Dias, C. R. G. (2019). The Fishing Productivity Assessement Upstream and Downstream of Tucuruí Hydroeletric Dam, Tocantins-Araguaia basin, Brazil. International Journal of Advanced Engineering Research and Science, 6(4):85-92.

Ruffino, M. L. (2004). Pesca e os recursos Pesqueiros na Amazônia brasileira. IBAMA/PróVárzea, Manaus-AM, 272pp.

Ruffino, M. L. (2008). Sistema integrado de estatística pesqueira para a amazônia. PanAmerican Journal of Aquatic Sciences, 3(3):193-204.

Zacarkim, C. E., Piana, P. A., Baumgartner, G., and Aranha, J. M. R. (2015). The panorama of artisanal fisheries of the Araguaia River, Brazil. Fisheries Science, (81):409416. 